\title{
Logarithmic Compression of Sensory Signals within the Dendritic Tree of a Collision-Sensitive Neuron
}

\author{
Peter W. Jones ${ }^{1}$ and Fabrizio Gabbiani ${ }^{1,2}$ \\ ${ }^{1}$ Department of Neuroscience, Baylor College of Medicine, Houston, Texas 77030, and ${ }^{2}$ Department of Computational and Applied Mathematics, Rice \\ University, Houston, Texas 77005
}

\begin{abstract}
Neurons in a variety of species, both vertebrate and invertebrate, encode the kinematics of objects approaching on a collision course through a time-varying firing rate profile that initially increases, then peaks, and eventually decays as collision becomes imminent. In this temporal profile, the peak firing rate signals when the approaching object's subtended size reaches an angular threshold, an event which has been related to the timing of escape behaviors. In a locust neuron called the lobula giant motion detector (LGMD), the biophysical basis of this angular threshold computation relies on a multiplicative combination of the object's angular size and speed, achieved through a logarithmic-exponential transform. To understand how this transform is implemented, we modeled the encoding of angular velocity along the pathway leading to the LGMD based on the experimentally determined activation pattern of its presynaptic neurons. These simulations show that the logarithmic transform of angular speed occurs between the synaptic conductances activated by the approaching object onto the LGMD's dendritic tree and its membrane potential at the spike initiation zone. Thus, we demonstrate an example of how a single neuron's dendritic tree implements a mathematical step in a neural computation important for natural behavior.
\end{abstract}

\section{Introduction}

Explaining how neural circuits implement specific computations on sensory stimuli relevant for behavior presents a central challenge for neuroscience. The task requires an understanding of the anatomy of neural circuits, their physiology, and their mapping onto specific building blocks of the investigated computation. Classic studies from this perspective include those related to the jamming avoidance behavior of weakly electric fish, directional motion selectivity in the vertebrate retina and visual cortex, as well as directional selectivity in the insect visual system (Heiligenberg, 1991; Priebe and Ferster, 2008; Jones and Gabbiani, 2010; Wei and Feller, 2011; Borst and Euler, 2011). Yet, we do not have a detailed understanding of how specific signals are represented in real time at successive stages of the neural networks involved in these computations.

More recently, a neural pathway in the locust visual system has been proposed as a model to study how a single neuron and its presynaptic network may implement multiplicative computations (Hatsopoulos et al., 1995; Koch, 1998). Multiplication lies at the heart of several algorithms implemented neurally, most famously the Reichardt correlation model of directional motion detection in insects (Hassenstein and Reichardt, 1956; Borst et al.,

Received Nov. 17, 2011; revised Feb. 8, 2012; accepted Feb. 11, 2012.

Author contributions: P.W.J. and F.G. designed research;P.W.J. performed research; P.W.J. analyzed data; P.W.J. and F.G. wrote the paper.

This work was supported through grants from the National Institute of Mental Health, the National Science Foundation, and a fellowship from the National Institute for Biomedical Imaging and Bioengineering.

Correspondence should be addressed to either of the following: Peter W. Jones, Center for the Neural Basis of Cognition, Carnegie Mellon University, 4400 Fifth Avenue, Pittsburgh, PA 15213, E-mail: pwjones@cnbc.cmu.edu; or Fabrizio Gabbiani, Department of Neuroscience, Baylor College of Medicine, One Baylor Plaza, Houston, TX77030, E-mail: gabbiani@bcm.edu.

DOI:10.1523/JNEUROSCI.5777-11.2012

Copyright $\odot 2012$ the authors $\quad 0270-6474 / 12 / 324923-12 \$ 15.00 / 0$
2010). In the locust, an identified neuron called the lobula giant movement detector (LGMD) lies at the apex of a converging sensory pathway specialized in detecting objects approaching on a collision course (O'Shea et al., 1974; Schlotterer, 1977; Rind and Simmons, 1992). This pathway has been extensively studied using looming stimuli, two-dimensional expanding shadows projected onto a screen to simulate approaching objects (Fotowat et al., 2011). These stimuli cause the firing rate of the LGMD to initially increase, then peak, and finally decay toward baseline late during the simulated approach. The peak firing rate always occurs a fixed delay after the time at which the looming stimulus reaches an angular threshold on the retina, independent of the stimulus' specific characteristics, such as the simulated object's size, speed, texture, or approach direction (Gabbiani et al., 1999; 2001). Neurons with nearly identical response profiles have been identified in a variety of vertebrate and invertebrate species (Sun and Frost, 1998; Wu et al., 2005; Preuss et al., 2006; Fotowat et al., 2009; Nakagawa and Hongjian, 2010; Liu et al., 2011).

How does the LGMD neuron implement this angular threshold computation? The LGMD receives $\sim 15,000$ cholinergic excitatory synapses whose activation is related to the speed of moving objects (Strausfeld and Nässel, 1981; Rind and Simmons, 1998; Krapp and Gabbiani, 2005). In addition, it receives $\sim 1000$ GABAergic inhibitory synapses, whose activation depends on stimulus size (Rowell et al., 1977; Gabbiani et al., 2005). The model that best explains the time course of the neuron's firing rate is essentially unique and multiplies the angular speed of the looming stimulus with a negative exponential of its size (see Fig. 1; Gabbiani et al., 1999). This suggests that feedforward excitation to the neuron encodes angular speed while feedforward inhibition mediates the size signal. Further data support the hypothesis that this multiplication is achieved within the neuron 
through addition of the excitatory and inhibitory inputs transformed logarithmically, followed by an approximate exponentiation, taking place at the spike initiation zone (Gabbiani et al., 2002). However, the quantitative relationship between the excitatory input to the LGMD and stimulus velocity has remained unknown, as has the relationship between the inhibitory input and angular size. Also missing is an understanding of how these signals are processed presynaptic to the LGMD. As a result, the specific computation carried out within the LGMD has remained unclear.

Recently, we characterized the encoding of stimulus speed in the retina and the neuropils presynaptic to the LGMD (Jones and Gabbiani, 2010). Based on these data, we simulated the activation of neural populations at successive stages of the pathway leading to the LGMD. We used the results to drive a compartmental model of the cell. Our simulations demonstrate that speed is encoded expansively by the excitatory neurons converging onto the LGMD and show the postulated logarithmic compression of angular speed to be implemented within the dendrites of the LGMD. The results provide predictions for future experiments and, more generally, have implications for the implementation of neural computations in other systems.

\section{Materials and Methods}

Electrophysiology. This report further analyzes data previously presented in Jones and Gabbiani (2010). Detailed methods of the recordings throughout the locust visual system can be found there. Briefly, as illustrated in Figure $1 A$, sharp-electrode intracellular recordings were carried out in adult locusts (Schistocerca americana) of either sex. Recordings were made from individual photoreceptors $(n=27$ cells), large monopolar cells (LMCs) in the lamina $(n=3)$, and from the LGMD in both voltage-clamp and current-clamp modes $(n=12$ and 22 facets in 6 animals, respectively).

Visual stimulation. Visual stimuli were generated using custom software on a personal computer running a real-time operating system (QNX 4, QNX Software Systems). Two apparatuses were used: a conventional cathode ray tube (CRT) monitor $(200 \mathrm{~Hz}$; luminance range, 2-90 $\mathrm{cd} / \mathrm{m}^{2}$ ) and a custom-designed projector-microscope system. The projector-microscope system enabled stimulation at single-ommatidium (facet) resolution. This was achieved by projecting an image generated using a digital light processing projector (LT140, NEC) through a custom-built microscope mounted horizontally on a vibration-isolated optical table (illuminance range, 4-2530 lux) (Jones and Gabbiani, 2010). Both the CRT and projector were calibrated to ensure linear, 6 bit resolution control over light levels. The ambient light level for single-facet experiments was set by a ringlight mounted around the objective of the microscope. This level was constant at 490 lux, equivalent to dim daylight. Locusts stimulated using the CRT monitor were adapted to a slightly different light level, determined by the brightness of the monitor (280 lux). In photoreceptors, the mean resting membrane potential $\left(V_{\mathrm{m}}\right)$ values recorded using single-facet and monitor stimulation were very similar: $-40.4 \mathrm{mV}$ and $-39.1 \mathrm{mV}$, respectively.

The CRT monitor was used to record the responses of photoreceptors to dark edges translating across their receptive fields over a broad range of speeds (see Fig. $2 A$ ). The slope of their hyperpolarizing responses were fit to a saturating function of Michaelis-Menten type as follows:

$$
S_{p h}(\psi)=\eta \psi /(\rho+\psi)
$$

where $\psi$ is the edge velocity, and $\eta$ and $\rho$ are fit parameters giving the saturation slope and the speed at half maximum slope, respectively. Fits to this equation as well as those to other functional forms listed below were quantified by computing $r^{2}$ values according to the formula $r^{2}=$ $1-S S_{\text {res }} / S S_{\text {dat }}$, where $S S_{\text {res }}=\Sigma\left[\text { slope }_{\mathrm{i}}-S_{\mathrm{ph}}\left(\psi_{\mathrm{i}}\right)\right]^{2}$ is the sum of squared residuals for the slope fit, and $S S_{\text {dat }}=\Sigma\left(\text { slope }_{i}-\text { mean }_{\text {slope }}\right)^{2}$ is the squared deviation of the data from its mean, $\operatorname{mean}_{\text {slope }}$. In these equations, slope $\mathrm{i}_{\mathrm{i}}$ denotes the $i$-th slope data point for edge velocity $\psi_{\mathrm{i}}$.
The CRT monitor was also used to record LGMD responses to looming stimuli, as previously described (Gabbiani et al., 1999). The looming stimuli were expanding dark squares on a bright background, as schematized in Figure $1 B$. Let $l$ denote the half-size of the simulated object, $v$ its simulated approach velocity, and let $t$ be time measured relative to projected collision. By convention, $v$ is negative for an approaching object, $t$ is $<0$ before collision ( $0=$ projected collision time), and, consequently, the time-varying distance from the observer is $v \cdot t$ (see Fig. $1 B$ ). If $\theta$ denotes the angular size of the square on the retina, then from the shaded right triangle in Figure $1 B, \tan (\theta / 2)=l / v t$. Inverting this equation yields $\theta(t)=2 \cdot \tan ^{-1}(l / v t)$. The angle, $\theta(t)$, is thus fully described by the half-size-to-speed ratio, $l|| v \mid$, with units of time. Assuming a constant simulated object size, the lower the $l /|v|$ value, the faster the object is approaching and the more suddenly it expands. The maximum $\theta$ value subtended at the eye by a stimulus on the monitor was $82^{\circ}$. The angular velocity, $\psi(t)=\theta^{\prime}(t) / 2$, of the expanding square's edges is given by the following equation:

$$
\psi(t)=\frac{-l / v}{t^{2}+(l / v)^{2}}
$$

The projector-microscope system was used to record the responses of single photoreceptors, LMCs, and the LGMD to a series of luminance changes from bright to dark (Jones and Gabbiani, 2010). These luminance changes had the time course of a cumulative Gaussian. Because the spatial receptive field of photoreceptors is approximately Gaussianshaped (Wilson, 1975), such a time course matches that of an edge sweeping across the receptive field at constant speed. More specifically, an edge traveling at a constant speed $\psi$ (in degrees per second), and sweeping through a Gaussian receptive field of width $\sigma_{x}$ (in degrees), will produce a local, time-dependent luminance change that has the functional form of a cumulative Gaussian with a duration determined by its $\mathrm{SD} \sigma_{\mathrm{t}}=\sigma_{\mathrm{x}} / \psi$ (in seconds). The luminance changes presented to individual facets were parameterized by the amount of time it took to pass from maximum to minimum luminance (luminance change duration), which equals $4 \cdot \sigma_{t}$. From photoreceptor recordings, we determined the relationship between the duration of luminance change and the slope of the evoked hyperpolarizing response of photoreceptors (see Fig. $2 B$ ). This relationship was fitted by the following version of Equation 1, which depended on luminance change duration:

$$
S_{p h}(L D)=\eta \sigma_{x} /\left(\frac{\rho L D}{4}+\sigma_{x}\right)
$$

where $L D$ is the luminance change duration of the stimulus and $\sigma_{x}$ is as defined above. Plotting the peak depolarization elicited by the same stimuli at later stages of the pathway against these photoreceptor slopes yielded functional relations fitted by Weibull functions of the form as follows:

$$
F(x)=\gamma \cdot\left(1-e^{-(x / \lambda)^{k}}\right)
$$

where $x$ is the photoreceptor response slope and $\gamma$ was constrained to 1 , since the peak responses were normalized (see Fig. $3 A$ ). The normalization for each experiment was performed by subtracting the mean spontaneous response level, then dividing each trial's response by the mean peak response to instantaneous luminance changes. Because photoreceptor recordings and those at later stages of the pathway were not performed simultaneously, they could not be directly compared. Thus, to take into account the variability observed in the data, we fitted the Weibull functions to Gaussian distributed surrogate data generated from the observed means and SDs for each recording condition (10,000 points per condition). To check that the functional description of Equation 4 did not miss any important aspect of the empirical relationship, we also ran the LGMD model simulations described below using a functional relationship between photoreceptor slope and normalized peak responses derived from a cubic spline fit to the mean data points. This yielded very similar results.

By combining the single-facet stimulation data recorded along the visual pathway with the photoreceptor responses to translating edges 
obtained with the CRT monitor, we were able to model the responses of individual LGMD inputs to looming stimuli, as explained next.

Modeling of inputs to the LGMD generated by looming stimuli. All data analysis and generation of model inputs was done using custom programs in Matlab (Mathworks). Simulations using a compartmental model of the LGMD were performed in the NEURON simulation environment (Hines and Carnevale, 1997). The compartmental model had active conductances similar to those of Peron et al. (2009). The model possesses realistic spike rate dynamics, with spike frequency adaptation governed by spike-triggered $\mathrm{Ca}^{2+}$ influx and a calcium-dependent potassium channel, as is observed in vivo (Peron and Gabbiani, 2009a). We computed the compartmental model's $V_{\mathrm{m}}$ and instantaneous firing rate (IFR) based on the excitatory and inhibitory synaptic conductances activated during a looming stimulus.

Simulated looming stimuli were generated as described above. For all simulations, visual space was discretized with a resolution of $0.1^{\circ}$ and spanned $82^{\circ}$ in azimuth and elevation (maximal looming square extent), centered in the middle of the eye at its equator, and $90^{\circ}$ from the front of the animal. The neural population of excitatory fibers presynaptic to the LGMD was simulated as a set of 2674 inputs spanning this $82 \times 82^{\circ}$ portion of the visual field. The simulated photoreceptors were distributed across visual space based on a complete reconstruction of the visual sampling pattern of a locust eye (Krapp and Gabbiani, 2005). Because no information is available on how photoreceptor receptive field sizes may vary across the locust eye, they were assumed to have a uniform angular size, defined by a $2 \mathrm{D}$ Gaussian with an $\mathrm{SD}$ of $3 / 4^{\circ}$. During a looming stimulus, the angular speed of an edge sweeping across the receptive field of a photoreceptor is continually increasing (Eq. 2; see Figs. $1 C, 5 B$ ). Hence, to bridge with the experimental data described above, we adopted the procedure summarized in Figure $4 A$ and explained in detail below. First, we fitted a cumulative Gaussian to the associated luminance change integrated over the photoreceptor receptive field. The optimal value of its $\mathrm{SD}, \sigma_{\mathrm{t}}$, yielded a corresponding "effective" edge speed over the photoreceptor receptive field from the relation $\psi_{\text {eff }}=\sigma_{t} / \sigma_{x}$. This effective edge speed enabled us in turn to read out from Figure $2 A$ the corresponding rate (slope) of hyperpolarization a real photoreceptor would have experienced in response to the stimulus. From this slope value we could then deduce, using Figure $3 A$, the associated peak response caused by such an input at later stages of the pathway, which consisted of LMCs, the LGMD's input current $\left(I_{\mathrm{m}}\right)$ and the LGMD $V_{\mathrm{m}}$. The combined mapping from edge speed to peak response is also illustrated in Figure 3B. This method treats photoreceptor response slope as a reliable bridge between two different stimulus conditions having different brightnesses, enabling us to avoid the assumption that response strengths under the two conditions are identical.

The second parameter used to simulate looming responses at each subsequent stage of the pathway was the latency associated with the luminance change duration that had stimulated its upstream photoreceptor (see Fig. 4A). As illustrated in Figure 3C, our data show a linear relationship between luminance change duration and the timing of the peak response at each level of the excitatory pathway (Jones and Gabbiani, 2010). To simulate neural responses to stimuli presented using the CRT monitor, we adjusted for slight differences in timing caused by the different brightness of the projector-microscope system with which we measured these timings. For this purpose, we measured the response latencies of photoreceptors using both stimulus displays (see Fig. 3C), calculated the differences between them, and adjusted the timings accordingly at each subsequent stage of the excitatory pathway (see Fig. 3D).

To obtain the time course of the compound excitatory stimulus at each stage of the model, we summed the inputs over the entire population at that stage in $2 \mathrm{~ms}$ bins and smoothed the resulting histogram with a Gaussian filter (SD, $8 \mathrm{~ms}$; see Fig. $5 \mathrm{C}$ ). This was performed based on input timings and magnitudes for the LMCs, the $I_{\mathrm{m}}$ in voltage clamp and $V_{\mathrm{m}}$ in current clamp (see Figs. $1 \mathrm{~A}, 3,5 \mathrm{~A}$ ). The excitatory synaptic input to the compartmental model of the LGMD was based on the input timing and magnitudes obtained at the stage of the LGMD $I_{\mathrm{m}}$. We created six synapses for each facet input, as expected based on physiological data (Peron et al., 2009). Their timing and magnitudes were given variability around their base value corresponding with that observed in vivo (Jones and Gabbiani, 2010). Each synapse was implemented as an $\alpha$ function conductance change with a maximum conductance of $0.54 \mu \mathrm{S}[\tau=0.3$ ms; reversal potential $\left(E_{\text {rev }}\right)=0 \mathrm{mV}$ ]. These parameters are consistent with those used for nicotinic acetylcholine synapses in other locust modeling studies (Bazhenov et al., 2001) and known receptor properties (van den Beukel et al., 1998). Depending on the effective edge speed of the stimulus, the magnitude of this conductance was scaled by the normalized response shown in Figure 3B. The dendritic location of each synapse was determined by the retinotopic location of its associated photoreceptor receptive field as shown in Figure $4 D$. The resulting time course of the total excitatory synaptic conductance, $g_{\text {exc }}$ (see Fig. $7 A$ ), was very similar to the normalized magnitude profile of the LGMD $I_{\mathrm{m}}$ (see Fig. $5 C$ ), except for a slightly shallower rise due to the introduced synaptic variability. To generate the inhibitory synaptic input to the LGMD, we triggered a set of four $\alpha$-conductance synapses at a constant delay of $70 \mathrm{~ms}$ after the luminance change over a photoreceptor receptive field had reached its midpoint $\left(g_{\max }=7.5 \times 10^{-3} \mu \mathrm{S} ; \tau=3 \mathrm{~ms} ; E_{\mathrm{rev}}=-75 \mathrm{mV}\right)$. These synapses were located on a small section of the main dendritic trunk where the inhibitory dendritic fields would connect in a complete anatomical model (see Fig. 4D). Each inhibitory synapse also had a constant amount of variability in magnitude and timing constrained by the experimental variability of the LGMD membrane potential (Jones and Gabbiani, 2012). The resulting total inhibitory input, $g_{\text {inh }}$, thus closely reflected the angular area covered by the stimulus, with a constant delay (see Fig. 7G).

The assumption that population responses are a summation of singlefacet responses is most likely a simplification. However, it has been shown that signals from two adjacent facets are largely independent of each other within the LGMD, regardless of their apparent motion delay (speed) and direction (Jones and Gabbiani, 2010). Previous experiments have shown spatially dependent inhibitory effects attributed to the circuitry presynaptic to the LGMD (O'Shea and Rowell, 1975), but their impact during looming is probably weak (Gabbiani et al., 2002, 2005). More complicated multifacet interactions may help shape signals reaching the LGMD in vivo (Peron et al., 2009); however, because they have not yet been identified, we have not incorporated them into the model.

Determining LGMD model parameters. The compartmental model of the LGMD used was constrained using a wealth of data collected in recent years. The model is based on the compartmental model used in Peron et al. (2009), which is an anatomical simplification of the reconstructionbased anatomy of the model in Peron et al. (2007). The passive parameters $\left(R_{\mathrm{a}}=60 \Omega \mathrm{cm}^{2} ; C_{\mathrm{m}}=1.5 \mathrm{mF} / \mathrm{cm}^{2}\right)$ were determined in Peron et al. (2007) and the value of $R_{\mathrm{m}}\left(10,350 \Omega \mathrm{cm}^{2} ; E_{\text {leak }}=-70 \mathrm{mV}\right)$ was set to produce an input resistance of $9 \mathrm{M} \Omega$, as observed in vivo with spontaneous activity blocked pharmacologically (Dewell and Gabbiani, unpublished observations). After we added spontaneous synaptic activity to produce spontaneous membrane variability as observed in vivo (Jones and Gabbiani, 2012), the final input resistance of the model was $4.5 \mathrm{M} \Omega$. The kinetics and concentrations of the spike-generating active conductances $\left(I_{\mathrm{Na}}\right.$ and $\left.I_{\mathrm{KDR}}\right)$ were adjusted to produce realistic spike waveforms and to match LGMD spiking responses to current injection (Peron and Gabbiani, 2009a). Conductance densities were constant in the area surrounding the spike initiation zone (SIZ; see Fig. $4 D, \mathrm{~K}_{\mathrm{Ca}}$ region; $I_{\mathrm{Na}}, 217$ $\mathrm{mS} / \mathrm{cm}^{2} ; I_{\mathrm{KDR}}, 353 \mathrm{mS} / \mathrm{cm}^{2}$ ) and were a quarter as dense in the axon. The model's voltage-gated $\mathrm{Ca}^{2+}$ channels, $\mathrm{K}_{\mathrm{Ca}}$ channels, and $\mathrm{Ca}^{2+}$ clearance mechanism controlled the model's spike frequency adaptation (SFA) and had densities and kinetics appropriate to recreate the SFA observed in vivo (Gabbiani and Krapp, 2006; Peron and Gabbiani, 2009b). The excitatory synaptic input strength to the LGMD was constrained by simulating single-facet stimulation trials as a short burst of spikes in the LGMD's presynaptic inputs (Peron et al., 2009; Jones and Gabbiani, 2012), resulting in six synapses firing three times each at $200 \mathrm{~Hz}$. The maximal excitatory synaptic conductance was adjusted so that the resulting compound EPSP had a mean amplitude of $\sim 10 \mathrm{mV}$ at the base of the dendritic tree, replicating a typical single-facet stimulation experiment (Jones and Gabbiani, 2010). The variability of the synaptic conductances (magnitude, jitter) was also set to replicate the variability observed in single-facet stimulation experiments. The strength of the inhibitory synaptic conduc- 
tance was determined as that which successfully produced appropriately timed peaked responses to looming stimuli with firing rates in the correct range. The pattern of inhibitory input, as described above, was based on available data and no attempt was made to change the time course of this input to produce realistic response profiles.

Speed-tuning functions. To quantify how the neural populations presynaptic to the LGMD and the LGMD itself encode looming stimulus velocity, we calculated the relationship between the dynamic velocity of the stimulus edge and the evoked response. These angular speed-tuning functions at each stage of the pathway are plotted in Figure 5D. To compute them, we needed to account for delays caused by neural latencies as signals propagate along the pathway. Therefore, each tuning function plots the instantaneous population response strength at time, $t$, versus the instantaneous stimulus angular speed, $\psi$, at time $t-\tau$. The latency parameter, $\tau$, was computed at each stage of the pathway individually. For each level, $\tau$ was calculated as the temporal offset that maximized the correlation between stimulus angular velocity and response. The optimal delay was computed for each $l /|v|$ separately, then averaged across $l /|v|$ to yield one value for each stage along the pathway. Resulting temporal offsets were as follows: $\tau_{\text {IFR }}=$ $93 \mathrm{~ms}, \tau_{\mathrm{Vm}}=86 \mathrm{~ms}, \tau_{\mathrm{Im}}=68 \mathrm{~ms}$, and $\tau_{\mathrm{LMC}}=$ $39 \mathrm{~ms}$; offsets for individual $l /|v|$ values varied by $\pm 2-5 \mathrm{~ms}$ around those means. Functionally, these offsets are several milliseconds longer than the minimum latencies introduced to single-facet signals in the model, as expected. This procedure enabled the speed-tuning functions to accurately capture how the velocity dependence of single-facet latencies (see Fig. 3D) shapes the velocity dependence of population responses.

To obtain a compact description of the shape of the resulting curves, we fit power-law functions to the speedtuning curves of the form $R(\psi)=a\left(\psi / \psi_{\max }\right)^{n}$. They were fit in the range between $2 \%$ and the velocity, $\psi_{\max }$, at which the stimulus reached the angular threshold size detected by the model LGMD's peak firing rate. Fitted parameter values are given in Figure 5. To ensure that the shape of the speed-tuning functions was not dependent on the exact value of the latency parameter $\tau$ used, we also fit several values between $0 \mathrm{~ms}$ and those stated above. For all $\tau$ values tried, speed-tuning functions were always well fit by power-law functions and the fitted $n$ was always $>1$. For smaller values of $\tau$, however, the fitted values of $a$ and $n$ decreased and the slopes of the curves for different $l /|v|$ values diverged: larger $l /|v|$ values, corresponding to slower approaches, had relatively steeper slopes. Thus, the value of $\tau$ influenced the steepness of the tuning curves but not their general shapes.

The Weibull functions fit to speed-tuning functions of $V_{\mathrm{m}}$ and the IFR in Figure 6 were of the same form as in Equation 2 above.

Spike threshold transformation. The nonlinear transformation occurring in spiking neurons in vivo between membrane potential and firing rate is important from the computational standpoint (Gabbiani et al., 2002; Peña and Konishi, 2002; Priebe et al., 2004). At the spike initiation zone of the LGMD, this transformation is known to be a power law approximating an exponential (Gabbiani et al., 2002). To characterize the equivalent transformation in the model, we computed the relationship between the model median-filtered $V_{\mathrm{m}}$ and the IFR in the following fashion, following the method used previously on experimental data
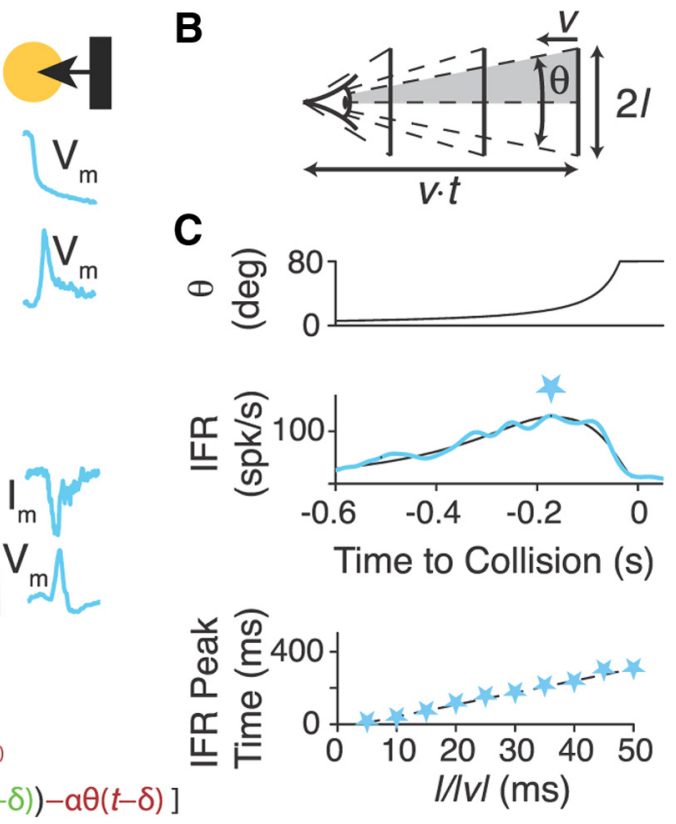

Med

Figure 1. Circuitry and physiology underlying a multiplicative neural computation in the locust optic lobe. $A$, Photoreceptors (Ph) hyperpolarize (right, cyan trace) as dark edges cross their receptive fields over single facets on the eye (top, yellow circles). Subsequently, that information propagates along an excitatory pathway comprising LMCs and medullary interneurons (Med)

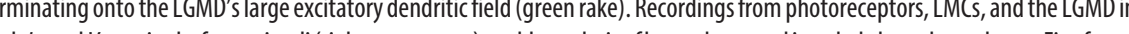

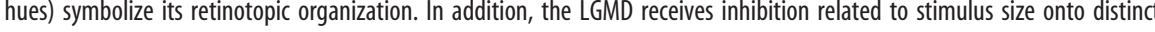
alf-size and vits approach velocity. The shaded right triangle is used to derive the time dependence of $\theta$ (see Materials and elation between peak time and $I /|v|$ is synonymous with the firing rate peak occurring a fixed delay, $\delta$, after the square reaches a ${ }^{-1}(1 / \alpha)$, over the animal's retina. ( adapted from Gabbiani et al. (2002).

(Gabbiani et al., 2002). Median-filtered $V_{\mathrm{m}}$ and IFR time series from all simulation runs were averaged in $5 \mathrm{~ms}$ bins spaced every $2.5 \mathrm{~ms}$. A regularly spaced $V_{\mathrm{m}}$ vector was created by pooling these values across time and binning them $\left(0.5 \mathrm{mV}\right.$ bins). The IFR values associated to each $V_{\mathrm{m}}$ bin were used to compute mean IFRs and their SDs. Threshold functions were fit using the method of least squares, minimizing the error function, as follows:

$$
\varepsilon^{2}=\sum_{i} \frac{\left(\mathrm{fit}_{i}-\mathrm{IFR}_{i}\right)^{2}}{\operatorname{IFR~} \mathrm{SD}_{i}^{2}}
$$

where $\mathrm{IFR}_{i}$ is the mean IFR in the $i$-th $V_{\mathrm{m}}$ bin and IFR $\mathrm{SD}_{i}$ its SD, while fit is the corresponding fitted value as a function of $V_{\mathrm{m}}$ in the $i$-th bin. The fitted function was $\operatorname{IFR}\left(V_{\mathrm{m}}\right)=\alpha \cdot\left(V_{m} / V_{\max }\right)^{n}$, where $V_{\max }(21.25 \mathrm{mV})$ is the maximal value of the membrane potential that was considered. The best power-law fit was for $\alpha=125$ and $n=1.57\left(r^{2}=0.95\right.$; see Fig. $\left.7 H\right)$. Code for reproducing the model and figures using model data is deposited on ModelDB (http://senselab.med.yale.edu/modeldb).

\section{Results}

Our goal was to understand how the neural populations presynaptic to the LGMD nonlinearly signal stimulus speed and how the LGMD integrates these inputs with size-dependent inputs to track looming stimuli over time. Specifically, the time course of 
A

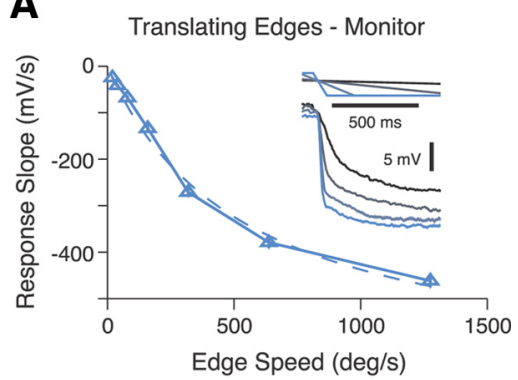

B Luminance changes - Projector

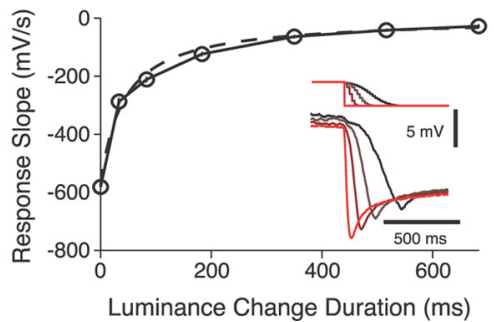

Figure 2. Photoreceptor responses to moving edges and luminance changes. $A$, Population average photoreceptor response slopes as a function of edge speed. The dashed line is a leastsquares fit to the data of a saturating function (Eq. 1; parameters: $\eta=-641 \mathrm{mV} / \mathrm{s}, \rho=$ $543^{\circ} / s, r^{2}=0.992$ ). Barely visible error bars show SEM. Inset shows representative photoreceptor responses (bottom traces) to dark translating edges (edge position on top; maximal displacement, $\left.102^{\circ}\right)$. Fast translations are in bright colors, with slower stimuli in darker colors $\left(20,80,319\right.$, and $1275^{\circ} /$, respectively). $\boldsymbol{B}$, Mean photoreceptor response slope to luminance changes as a function of luminance change duration. Dashed line shows best fit relationship (Eq. 3; $\left.\eta=-575 \mathrm{mV} / \mathrm{s}, \rho=73^{\circ} / \mathrm{s}, r^{2}=0.992\right)$. Inset shows representative photoreceptor responses to luminance changes of varying duration. Bright colors show faster luminance changes; darker colors are slower $(0,83,183,350 \mathrm{~ms})$. Top inset traces show the stimulus luminance over time. Differences in response transients between $\boldsymbol{A}$ and $\boldsymbol{B}$ are due to differences in ambient lighting and stimulus brightness but do not affect the range of photoreceptor response slopes (see Materials and Methods; Jones and Gabbiani, 2010).

the LGMD firing rate has been described as follows by a product of angular speed, $\psi(t)$, and a negative exponential of size, $\theta(t)$,

$$
f(t)=g\left(\psi(t-\delta) e^{-\alpha \theta(t-\delta)}\right)
$$

resulting in the detection of an angular threshold in the LGMD's peak firing rate (Fig. 1). In this equation, $g$ is a static (timeindependent) nonlinearity, the parameter $\alpha$ is related to the threshold angle detected by the peak firing rate and $\delta$ represents a neuronal delay (Gabbiani et al., 1999). A key aspect of this neural computation is how angular speed is encoded within the LGMD's excitatory input pathway. The approach we took to address this question was to characterize the coding of angular speed along the pathway using single-facet stimuli.

\section{Single-facet speed tuning}

The activation of the excitatory inputs to the LGMD by looming stimuli is highly synchronized and changing over time (Jones and Gabbiani, 2010). To obtain a precise quantitative description, we started by systematically documenting the response of single photoreceptors as a function of edge speed. The inset of Figure $2 \mathrm{~A}$ shows the membrane potential of a photoreceptor, recorded intracellularly, to translating bright-to-dark edges presented using a CRT monitor. As the edges traverse the cell's receptive field, the light level experienced by the receptor decreases and the membrane potential of the cell drops, normally $\sim 10-15 \mathrm{mV}$ (Jones and Gabbiani, 2010). We recorded photoreceptor responses to translating edges and computed their slope (Fig. 2A).
A
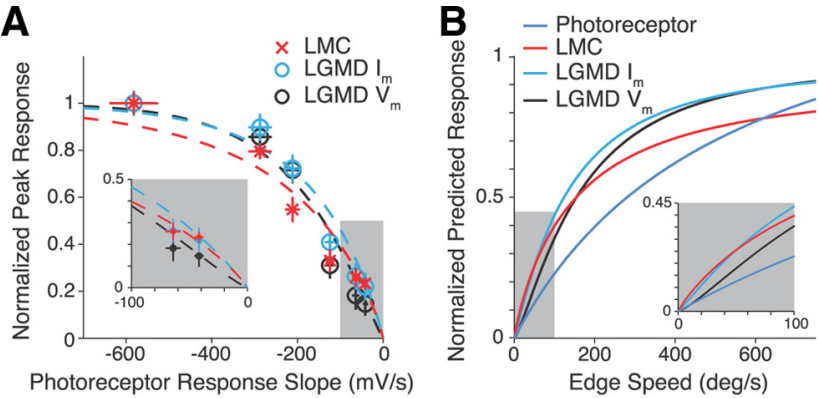

C

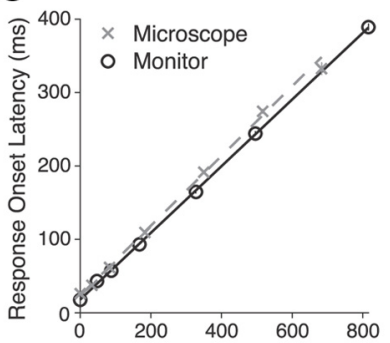

Luminance Change Duration (ms)

D

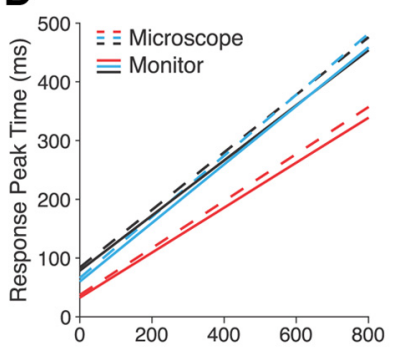

Luminance Change Duration (ms)

Figure 3. Single-facet speed tuning and response timing. $A$, The relationship between photoreceptor response slope and normalized response peak height is plotted for LMCs (red), the $\mathrm{LGMD} I_{\mathrm{m}}$ (cyan), and the LGMD $V_{\mathrm{m}}$ (black). Each point is the population average response for one luminance change duration (error bars give SEM). Dashed lines show Weibull function fits to the data (Eq. 4). The inset (gray shading) better shows the lower response portion of the data and fits. Typical normalization values: $\mathrm{LMCs}, 4.84 \mathrm{mV} ; \mathrm{LGMD} I_{\mathrm{m}^{\prime}}-1.75 \mathrm{nA}$; LGMD $V_{\mathrm{m}^{\prime}}, 6.6 \mathrm{mV}$. Fit parameters: LMC $\lambda=221.7 \mathrm{mV} / \mathrm{ms}, \kappa=1.11 ; \mathrm{LGMD} V_{\mathrm{m}} \lambda=172.7 \mathrm{mV} / \mathrm{ms}, \kappa=1.25 ; \mathrm{LGMD}$ $I_{\mathrm{m}} \lambda=193.7 \mathrm{mV} / \mathrm{ms}, \kappa=1.61$. Fit $r^{2}$ : LMCs 0.91; LGMD $V_{\mathrm{m}} 0.92 ; \mathrm{LGMD} I_{\mathrm{m}} 0.94$. B, Using the fits shown in $A$ and the relationship between stimulus speed and photoreceptor slope shown in Figure $2 A$, we calculate the single-facet speed tuning for photoreceptors, LMCs, and the LGMD $I_{\mathrm{m}}$ and $V_{\mathrm{m}}$. Coloring is as in $A$, and the inset shows the predicted responses to speeds up to $100 \%$. C, Adjusting response timings for display type. Response latencies of photoreceptors for wide-field luminance changes on the monitor (black) and single-facet luminance changes delivered with the projector-microscope (solid gray) are shown. Lines shown are the best fit linear functions, dashed and solid for the two conditions. $\boldsymbol{D}$, Conversions of response peak times through the visual pathway. Dashed lines show the linear fits to the timings of the peak responses for LMCs and the LGMD based on stimuli presented using the projector-microscope. Solid lines show the predicted peak times for stimuli presented using a monitor, the condition to be simulated. Adjustments shift latencies by $5 \mathrm{~ms}$ with an $\sim 4 \%$ change in slope of the relationship. Resulting parameters were slope $=0.38,0.50$, and 0.47 ; and intercept $=32.4,60.1$, and $78.4 \mathrm{~ms}$ for $\mathrm{LMC}, \mathrm{LGMD} I_{\mathrm{m}}$, and LGMD $V_{\mathrm{m}}$ respectively. Colors show recording type as in $\boldsymbol{A}$.

Because LMCs in the lamina, the next stage of the visual pathway, high-pass filter their inputs (Laughlin and Hardie, 1978; Juusola et al., 1995), the slope of photoreceptor hyperpolarization will have a major influence on downstream LMC responses. The relationship between edge speed and response slope was well fit by a simple saturating function (Eq. 1). To examine signals downstream of photoreceptors, we used light stimuli precisely delivered to single facets and having the time course of cumulative Gaussians. These stimuli were designed to mimic the light changes produced by edges moving through the Gaussianshaped receptive fields of photoreceptors. Photoreceptor membrane potential responses to these stimuli are shown in the inset of Figure $2 B$. We used stimulus luminance changes spanning a wide range of rates (parameterized by the duration of the luminance change) to mimic a range of edge speeds occurring during looming stimuli, and show in Figure $2 B$ the relationship between luminance change duration and photoreceptor response slope for the population of recorded photoreceptors $(n=17)$.

Next, we obtained intracellular recordings further along the LGMD visual pathway, including both current-clamp and 
A

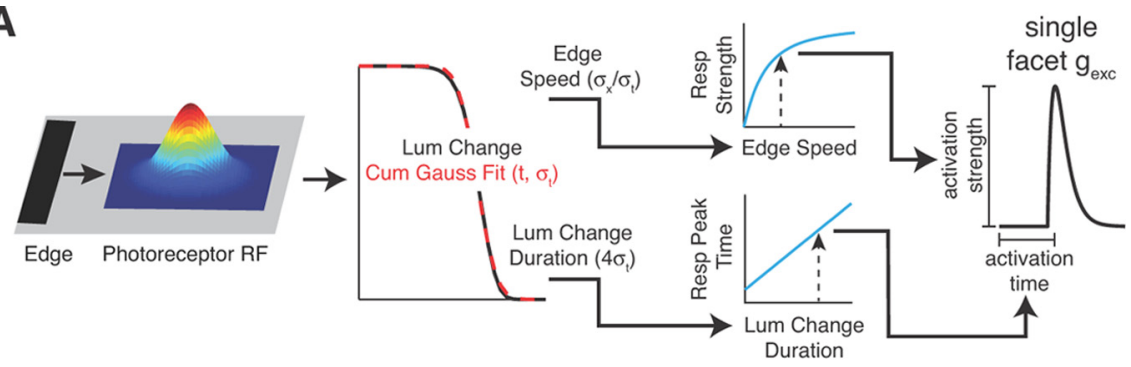

B

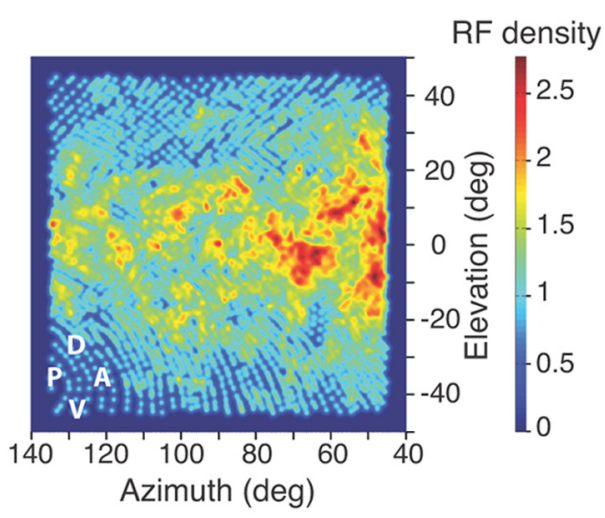

C

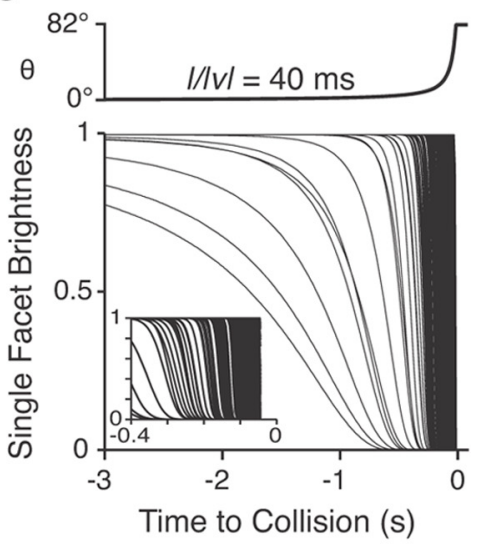

D

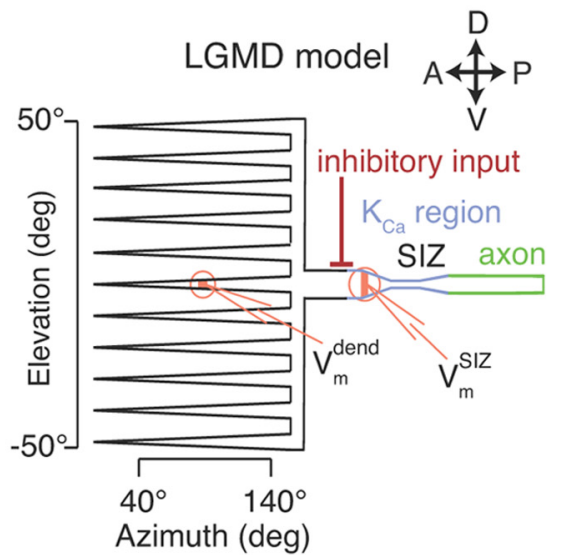

E

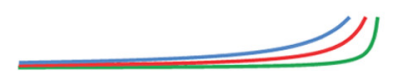

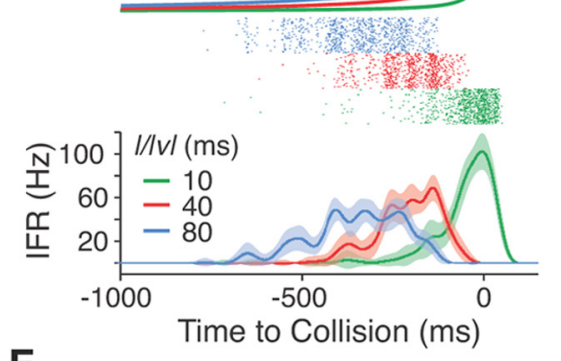

$\mathbf{F}$

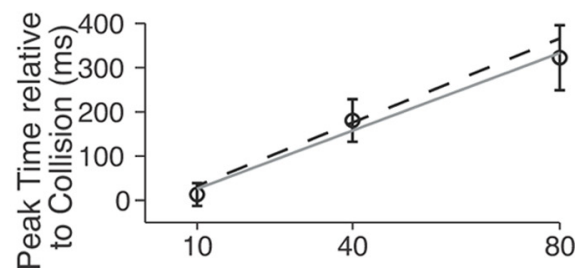

Figure 4. Model structure. Each LGMD compartmental model input was driven by a luminance change within the receptive field (RF) of its associated photoreceptor during a looming stimulus. The 2D Gaussian photoreceptor RFs were distributed across visual space based on a complete reconstruction of the optical sampling by a locust eye and had a uniform size (SD, $\left.\sigma_{\mathrm{x}}=3 / 4^{\circ}\right)$. $\boldsymbol{A}$, Schematic diagram illustrating how the strength of an individual synaptic input associated with a single photoreceptor was determined in the model. The luminance change caused by the looming stimulus edge crossing the photoreceptor's RF was fitted to a cumulative Gaussian. This yielded an equivalent edge speed that determined the peak synaptic conductance activation (top; see Fig. $3 B$ ). The duration of the luminance change is used to determine the latency of response through the relationships shown in Figure 3D (bottom). $\boldsymbol{B}$, Sampling density across the visual field, obtained by summing the weights of individual RFs at each location. Hot colors indicate more densely sampled regions of visual space. The maximum weight, for display purposes, of each RF is 1 , so the RF density gives the number of RFs sampling that area, if the RFs were perfectly overlapping. Receptive fields outside the maximum angular extent of the stimulus were omitted from the plot and the model. $\boldsymbol{C}$, The luminance input as a function of time to collision for simulated photoreceptors during the presentation of a looming stimulus. Each simulated photoreceptor integrated the stimulus luminance within its RF. During a looming stimulus, the luminance within the RF dropped. The top trace shows the angular size of the looming stimulus, while bottom traces show resulting luminance changes at individual RFs. The large number of RFs experiencing luminance changes shortly before collision causes the solid black in the $\sim 400 \mathrm{~ms}$ before collision. The inset expands the final $400 \mathrm{~ms}$ before projected collision. $D$, Schematic of the active compartmental model of the LGMD used. Excitatory input impinges retinotopically onto the rake-shaped dendritic tree and inhibitory synapses are made onto the dendritic segment immediately proximal to the rake. The model is modified from the one used in Peron et al. (2009). Compartments from which the dendritic and SIZ $V_{\mathrm{m}}$ were recorded are highlighted in orange. $\boldsymbol{E}$, Spiking responses of LGMD model to looming stimuli. Top traces

voltage-clamp recordings from the LGMD itself, and current-clamp recordings from large monopolar cells. Because all responses to single-facet luminance changes were acquired under identical conditions, they enabled us to directly compare response strengths at different stages of the pathway to those of photoreceptors. These relationships are shown in Figure $3 A$ and were well fit by Weibull functions (Eq. 4). From these empirical relationships and that shown in Figure $2 B$, we could extrapolate the single-facet speed tuning by transforming from stimulus speed to photoreceptor slope and from photoreceptor slope to response strength for each recorded stage of the pathway. The resulting speed-tuning functions are shown in Figure 3B.

We were also able to carry out a similar extrapolation for another important parameter of single-facet responses: their latencies. Previously, we showed that response latency increases linearly with luminance change duration (Jones and Gabbiani, 2010). Because we wish to simulate the responses of looming stimuli presented using a CRT monitor, here we must make a small adjustment in those measured relationships to account for different stimulus sources. Thus, photoreceptor response onset latencies to luminance changes were measured both with the single-facet projector-microscope (which we used for stimulation of all other cell types) and with the CRT monitor (on which we presented looming stimuli to the LGMD). These two modes of stimulation evoked responses of slightly different latencies (Fig. 3C), and we used that slight difference to adjust the response latencies of other cell types to stimuli presented with the CRT monitor (Fig. 3D).

\section{Simulation of wide-field looming responses}

The results described above can be used to simulate the activation of single photoreceptors by the edge of a looming stimulus crossing their receptive field, as well as the

$\leftarrow$

show the stimulus angular size for $I /|V|=10,40$, and $80 \mathrm{~ms}$. The rasters show spiking in 50 simulated presentations for each $I /|v|$ value, and bottom traces show the mean instantaneous firing rates of the model computed by convolution of individual spike trains with a Gaussian filter (SD, $20 \mathrm{~ms}$ ). Envelopes show SEM. $F$, The timing of the model LGMD peak firing rate (circles, error bars show SEM) depends linearly on $I /|v|$, with a best fit slope $\alpha=4.8$ and an intercept $\delta=14.7 \mathrm{~ms}$ (black dashed line; see Eq. 6). This corresponds with the peak response occurring $14.7 \mathrm{~ms}$ after the stimulus has reached a constant angular size of $23.7^{\circ}$. The gray line shows the best fit linear relationship found in Jones and Gabbiani (2010). 
A
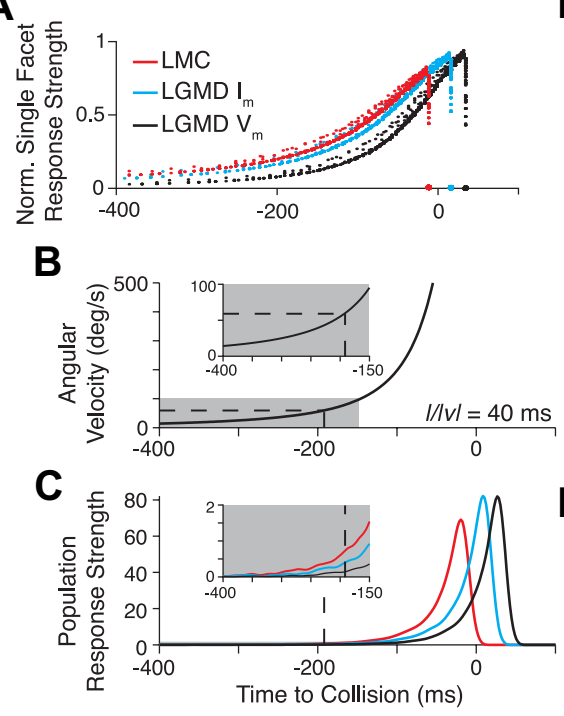

D

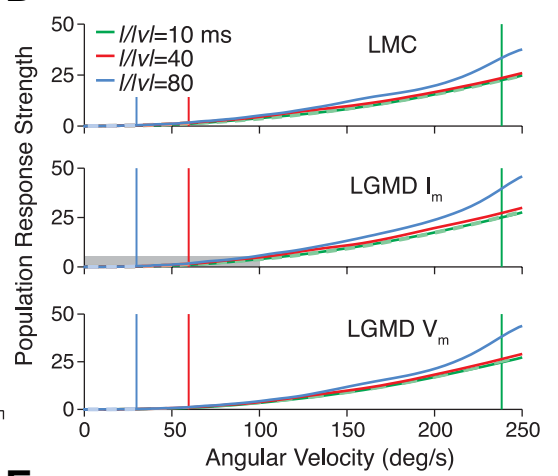

E

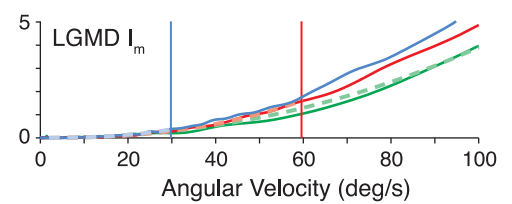

Figure 5. Dynamic population speed tuning to looming stimuli. $\boldsymbol{A}$, Strength of individual inputs over the course of a looming stimulus $(I /|v|=40 \mathrm{~ms})$ at each stage in the pathway. Each point is the normalized strength of an individual input, plotted at its time of activation (Fig. $4 A$ ). The color denotes the stage for which the timing and magnitude information was computed. $\boldsymbol{B}$, The angular velocity of the looming stimulus over time. Dashed lines show the average time and velocity at which the LGMD model responses reach their firing rate peak. C, The time-binned, summed, population input strength for each model stage (LMC, LGMD $I_{m}$ and LGMD $V_{m}$ ). The sum is taken over single-facet normalized inputs and smoothed as explained in Materials and Methods. Inset shows the sections of the plots leading up to the angular threshold time and velocity (light gray). $\boldsymbol{D}$, The strength of the excitatory input population is plotted against instantaneous angular velocity for different values of the looming stimulus parameter, $I /|v|$. Thin vertical lines show the stimulus velocities corresponding to angular threshold size for each stimulus. This velocity is different for different values of $I /|v|$. The plots show the tuning at successive stages of the pathway (LMC, $L G M D I_{m}$, and $\left.L G M D V_{m}\right)$. Lighter colored dashed lines show power-law fits to relationships ( $L M C: \alpha=22.46,1.48,0.38 ; n=2.10,2.17,2.30 ; r^{2}=0.999$, $0.997,0.980$ for $/ /|V|=10,40,80 \mathrm{~ms}$. LGMD $I_{\mathrm{m}}: \alpha=25.23,1.58,0.36 ; n=2.16,2.44,2.54 ; r^{2}=0.999,0.998,0.981 ; \mathrm{LGMD} V_{\mathrm{m}}$ : $\left.\alpha=24.92,1.07,0.19 ; n=2.28,2.67,2.79 ; r^{2}=0.999,0.998,0.983 ; \psi_{\max }=238.34,59.58,29.79^{\circ} / \mathrm{s}\right) . E$, LGMD $I_{\mathrm{m}}$ speed-tuning curves for velocities $<100 \%$ (detailed view of gray shaded area in $\boldsymbol{D}$ ).

resulting synaptic activation of the LGMD neuron and other neurons downstream of photoreceptors (Fig. 4A). Specifically, the integrated photoreceptor luminance change determines an equivalent edge speed and luminance change duration that yield corresponding peak synaptic strengths and activation timings, respectively. To simulate looming responses across an array of photoreceptors, we used a reconstructed map of their visual sampling directions across an entire locust eye (Krapp and Gabbiani, 2005). In this map, the sampling density varies widely across visual space, with a relatively dense stripe along the visual equator. The equatorial density peaks in front of the animal, outside of the region where our looming stimuli were simulated. Each photoreceptor receptive field was modeled by a symmetric 2D Gaussian with a full width of $3^{\circ}$ (Wilson, 1975). The resulting spatial weighting of visual information is illustrated in Figure $4 B$ by means of a "summed" receptive field strength.

We calculated the compound activation dynamics generated by a looming square in the population of neurons presynaptic to the LGMD from the description of the light changes taking place at each facet (Fig. 4A). The resulting signals are shown in Figure $4 C$. Because the square expands slowly at first, the photoreceptor light level initially decreases relatively slowly as it takes a long time for the stimulus to cover a receptive field. As the stimulus speeds up, light changes occur faster and include more facets. This results both from the stimulus edges moving more quickly and from the increasingly larger edges encountering more photoreceptor receptive fields. As described below, we used this pattern of light changes to examine the timing and strength of population activity at each stage of the LGMD pathway based on the single facet responses recorded from each cell type. We also used these population responses as the basis for synaptic input onto a compartmental model of the LGMD, illustrated in Figure 4D. Based on these inputs, the compartmental model produces sustained firing to looming stimuli, which ramps up until coming to a peak some time before collision (Fig. $4 E$ ). Like in vivo, these peak times are linearly related to the approach parameter characterizing the looming stimulus, defined as the half-size-to-speed ratio of the simulated approaching object, $l /|v|$ (Fig. $1 B$; see Materials and Methods for more details on the looming stimuli). These peak times also become more variable with larger $l /|v|$ values, again replicating in vivo results (Fig. $4 F$ ). Thus, the model was able to reproduce, within its error bounds, the main features of experimental LGMD responses to looming stimuli, although not all experimental features were fully accounted for. The membrane potential and spike train variability in response to looming stimuli were, for instance, typically higher in the experimental data than in the model (Jones and Gabbiani, 2012).

\section{Excitatory input responses to looming stimuli}

Looming stimuli are dynamic and vary greatly in angular size and speed during a simulated approach. Since the timing of LGMD activation to looming stimuli is a critical feature of its response, we examined the predicted activation time course of its inputs. Figure $5 \mathrm{~A}$ shows the relative magnitudes and peak response times at successive stages of the pathway leading to the LGMD. Each point in this plot corresponds to a response generated from the light change occurring at a single facet. The summed strength of these inputs over time is plotted in Figure $5 C$, while the time course of the looming stimulus angular velocity is shown in Figure 5B. Because latency increases with each stage, the activity climbs progressively later during the approach. The dashed lines on $5 B$ and $5 C$ mark the times and velocities at which the looming object's angular threshold is reached, only a few milliseconds before the simulated LGMD response would peak to such a stimulus $\left(23.7^{\circ}\right.$; Fig. $\left.4 F\right)$. Thus, the population responses preceding and up to those lines are most important for the LGMD's angular threshold computation. It is notable how small a proportion of the excitation occurs before this time. It should also be noted that the increase in the number of facets activated over time, combined with the increase in the magnitude of single-facet responses, causes the overall amount of excitation in the summed population to climb more steeply than the response strength from individual facets (Fig. $5 A, C$ ).

From these data we can compute speed-tuning curves along the pathway by plotting the instantaneous relationship between population activation at each stage and the angular velocity that evoked it. What is noticeable immediately is that these functions are expansive: response magnitude increases nonlinearly with stimulus velocity during the approach. In fact, they are well fit by power-law functions, with exponents ranging from $\sim 2$ to 3 (Fig. 
$5 D, E$, dashed lines). The overall steepness of these functions varies only slightly with either stimulus value or processing stage. Slower approaches (higher $l /|v|$ values) tend to have slightly steeper tuning functions than faster approaches, and the tuning function derived for the LGMD membrane potential is slightly shallower than those for the LMC stage and LGMD membrane current. The steepness also depends on the exact temporal offset used in the analysis to compensate for latency (see Materials and Methods for details), but the curves always retain their power-law shape, well described with exponents $>1$.

\section{Modeling of LGMD responses}

We next used the modeled input timings and strengths to drive the synaptic input to a compartmental model of the LGMD (Fig. $4 D$ ). This enabled us to determine how the angular speed tuning of LGMD inputs is altered by integration within the neuron and its relation to LGMD responses observed in vivo. Our compartmental model has an extended, but simplified, rake-like dendritic tree, where it receives excitatory synaptic inputs. It also possesses active conductances implementing realistic spike-rate adaptation. The inputs synapse retinotopically onto the dendritic tree, with frontal regions of the visual field activating distal synapses, and posterior regions activating proximal ones, while elevation is mapped across the width of the tree. In addition to the timevarying excitatory input described above, we also included inhibitory inputs stimulated with a fixed magnitude and delay after the stimulus reached each new facet (see Materials and Methods). Thus, synaptic inhibition was proportional to the area of the looming square, consistent with previous descriptions of feedforward inhibition onto the LGMD (Hatsopoulos et al., 1995; Gabbiani et al., 2005). Finally, synaptic noise in the model was adjusted to fit the variability of experimental data to single facet stimulation (Jones and Gabbiani, 2012).

We initially verified that the model displayed looming responses similar to those observed in vivo (Fig. $4 E, F$ ). Next, we ran simulations using only the excitatory inputs elicited by looming stimuli to compare the model's output with our calculated population inputs. The resulting angular speed tuning functions are shown in Figure 6 . Both the $V_{\mathrm{m}}$, taken from a compartment adjacent to the SIZ, and the IFR were normalized to their maximum across all $l /|v|$ values ( $41.7 \mathrm{mV}$ and $199 \mathrm{~Hz}$, respectively). In both cases, the model output starts to saturate before the angular speed at which the LGMD response would normally peak. All tuning curves are well fit by Weibull functions, shown as dashed lines (Eq. 4). For comparison, the fits to the speed tuning of the $V_{\mathrm{m}}$ summed input stage, taken from Figure $5 D$, are plotted as dotted lines in Figure 6A. Clearly, the angular velocity tuning at the output of the compartmental model has a very different shape than the summed membrane potential depolarization elicited by individual inputs to the LGMD.

\section{Biophysics of multiplicative computation within the LGMD}

The observed difference in velocity tuning of the compartmental model's responses and the excitatory input suggests that the excitatory input is substantially transformed within the LGMD neuron. This raises two questions about the underlying transformation: (1) where is the transformation localized in the neuron? and (2) is the transformation of velocity tuning related to the previously proposed scheme for the computation performed by the LGMD?

As to the first question, the transformation of velocity tuning could occur, in principle, within the excitatory dendrites of the model or between the excitatory dendrites and the spike initia-
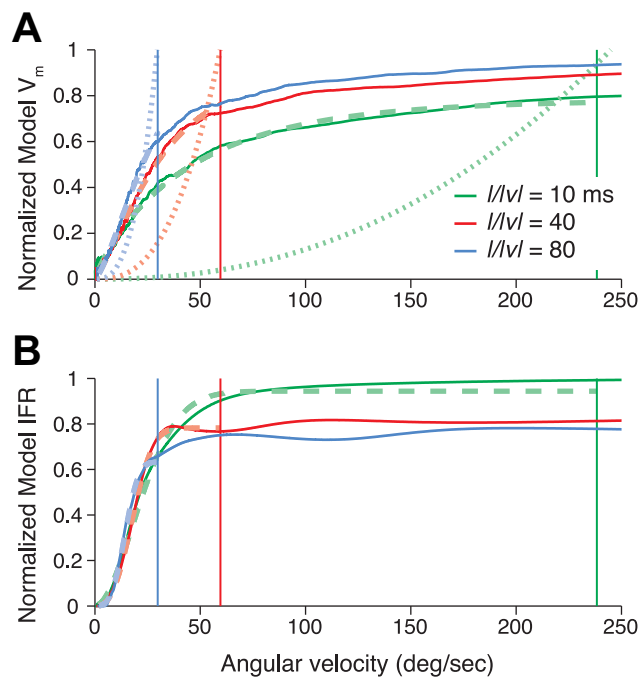

Figure 6. LGMD model output speed tuning. $A$, Speed-tuning functions calculated from the normalized $V_{m}$ of the compartmental model, obtained from a compartment near the model SIZ. Solid lines are the compartmental model speed-tuning curves. Lighter dashed lines are Weibull function fits, and dotted lines are fits from the normalized input population speed-tuning curves (see Fig. 5D), for comparison. Input population curves are normalized to their value at the angular threshold velocity (marked by vertical lines). $V_{m}$ is normalized to the maximum response across $/ /|v|$ values $(41.7 \mathrm{mV})$. Parameters for the fitted functions were $\gamma=0.78,0.87$, $1.01 \mathrm{mV}, \lambda=45.37,32.67,29.93(\mathrm{~s})$; and $\kappa=0.89,1.24,1.38, r^{2}=0.990,0.996,0.994$ for $I /|v|=10,40,80 \mathrm{~ms}$, respectively. $\boldsymbol{B}$, Speed tuning of the model IFR, formatted as in $\boldsymbol{A}$, normalized to $199 \mathrm{~Hz}$. Parameters of the fitted Weibull functions were $\gamma=0.94,0.78,0.63$, $\lambda=26.88,20.55,15.97(\%)$, and $\kappa=1.86,2.76,3.25, r^{2}=0.995,0.999,0.997$.

tion zone, given the extended electrotonic structure of the neuron and the location of the feedforward inhibitory input. Alternatively, the transformation could be due to the active conductances mediating spiking and spike frequency adaptation at the spike initiation zone. The latter possibility can be discarded since the shape of the model $V_{\mathrm{m}}$ and IFR in Figure 6 are qualitatively similar, and since pharmacological block of spike frequency adaptation has a minimal effect on the LGMD's responses to looming stimuli (Peron and Gabbiani, 2009a).

As to the second question, the previously proposed scheme for the computation performed by the LGMD is based on excitation being logarithmically related to angular velocity and added to inhibition signaling angular size, followed by an approximate exponentiation at the spike initiation zone (Gabbiani et al., 2002). Together, these steps functionally result in a multiplication of the excitatory and inhibitory inputs to the LGMD, as summarized by the following variant of Equation 6:

$$
f(t)=g[\exp [\log \psi(t-\delta)-\alpha \theta(t-\delta)]] .
$$

Our data and simulations enabled us to address this question within the biophysical model of the LGMD by examining how signals at different levels of the model and in different compartments of the neuron are interrelated and how they are related to the angular speed and size of the looming stimulus.

As a first step in evaluating the signal transformations in the context of the proposed computation (Eq. 7), we quantified the relationship between angular velocity and the total excitatory synaptic conductance to the LGMD model. Figure 7A shows the time course of these two signals, both nonlinearly increasing during the approach, and Figure $7 B$ shows the relationship between the two. The excitatory synaptic conductance is not linearly related to stimulus velocity, but well fitted by a power-law relationship with a different slope for each $l /|v|$ value. This mirrors the 
A

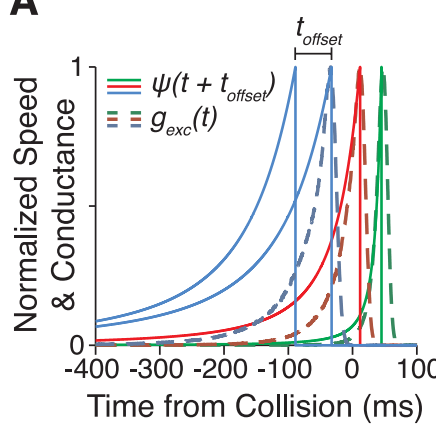

C

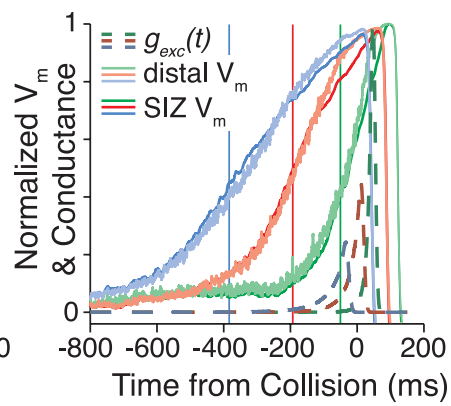

E

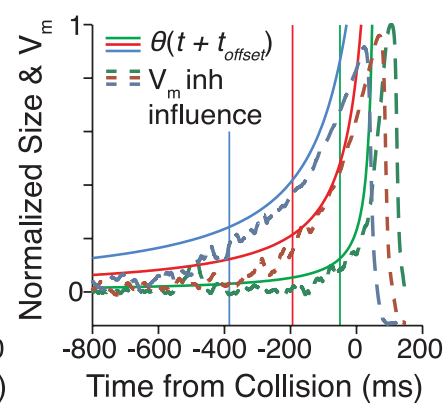

G

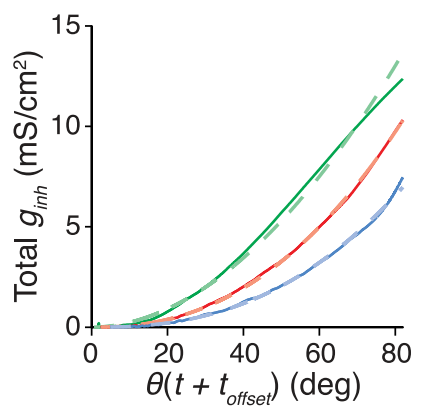

B

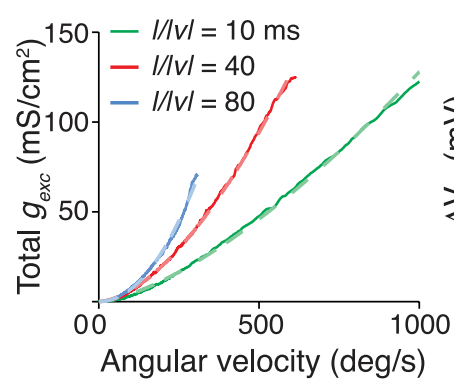

D

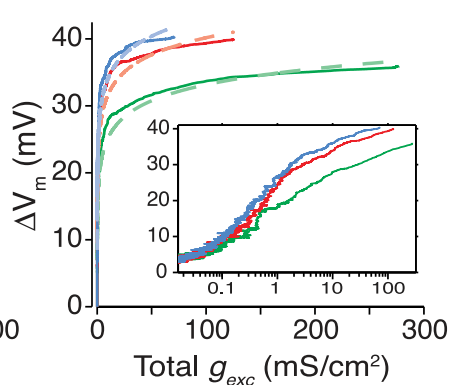

$F$

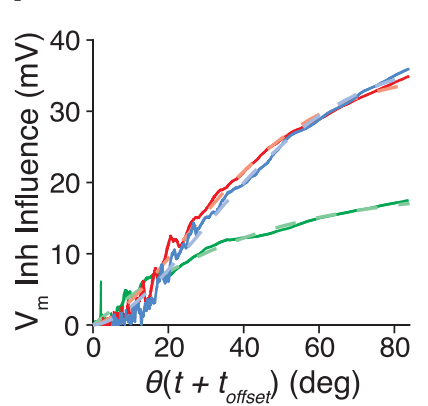

H

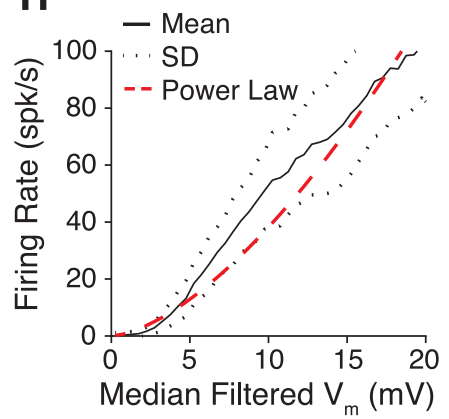

Figure 7. Transformation of angular speed and size inputs within the LGMD model. $\boldsymbol{A}$, The time course of angular velocity (solid lines) and total excitatory synaptic input ( $g_{\text {exc }}$ dashed lines) for $3 / /|v|$ values $(10,40,80 \mathrm{~ms})$. The blue angular velocity trace is shown before and after applying a time shift, $t_{\text {offset, }}$ to maximize its correlation with $g_{\text {exc }}$, whereas the others are shown only after temporal shifting. Time courses were normalized by their maximum value for each $/ /|v|$ value. Line colors in all panels denote $/ /|v|$ values, as in previous figures. $\boldsymbol{B}$, The relationship between angular velocity and total excitatory synaptic conductance. The quantity $g_{\text {exc }}(t)$ was plotted against $\psi\left(t+t_{\text {offset }}\right)$ where $t_{\text {offset }}(57$ ms) was chosen to be the mean value of temporal offsets for which there was the highest correlation with $g_{\text {exc }}(t)$ for each $/ /|v|$. Solid bright lines show this relationship for each $/ /|v| v a l u e$. Lighter dashed lines show power-law fits to this relationship. Fitted parameters are $n=1.48,1.68,2.05 ; \alpha=127.87,133.43,68.97 \mathrm{mS} / \mathrm{cm}^{2}$; with $\psi_{\max }=1000,641,321^{\circ} / \mathrm{s}, r^{2}=0.999,0.999,0.998$ for $/ /|v|$ values of 10,40 and $80 \mathrm{~ms}$, respectively. C, Membrane potential over time in multiple locations. Lighter traces show the membrane potential over time in a dendritic compartment corresponding to the center of the visual field (highlighted in Fig. 4D), while the brighter solid traces are the $V_{\mathrm{m}}$ near the SIZ. For comparison, the time course of $g_{\text {exc }}$ shown in $\boldsymbol{A}$, is replotted. $\boldsymbol{D}$, The relationship between $g_{\text {exc }}(t)$ and the resulting membrane potential near the SIZ. Colors show responses to different looming stimuli as in $A$. Solid lines show the observed relationship while dashed, lighter lines show fitted functions of the form $V_{m}=A$ log $\left(B g_{\text {exc }}\right)$. The inset shows the data on a logarithmic $x$-axis, demonstrating that the relationship is approximately linear. Fitted parameters are $A=3.46,3.68,3.91 \mathrm{mV}$ and $B=143.88,540.62$ and $612.79 \times 10^{-3}\left(\mathrm{mS} / \mathrm{cm}^{2}\right)^{-1}$, $r^{2}=0.85,0.76,0.75$ for $/ /|v|$ values of 10,40 , and $80 \mathrm{~ms}$, respectively. $\boldsymbol{E}$, Time course of the effect of inhibition is shown, plotted with the angular size of the stimulus. Signals are all normalized to their maximum across $/ /|V|$ values. The angular size trace is shifted by the same temporal offset as in $\boldsymbol{A}$ and $\boldsymbol{B}$. $\boldsymbol{F}$, The effect of inhibition on model $V_{\mathrm{m}}$ as a function of angular size. Solid lines show the

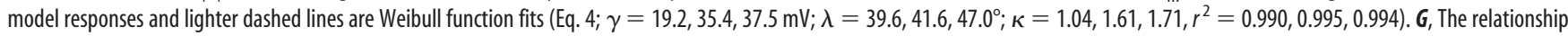
between angular size and the total inhibitory synaptic conductance. Lighter dashed lines show power-law fits $\left(n=1.92,2.32,2.47\right.$ and $\alpha=13.65,10.30,6.97 \mathrm{mS} / \mathrm{cm}^{2}$ with $\theta_{\text {max }}=82^{\circ}, r^{2}=$ $0.994,0.999,0.999) . H$, Spike threshold function for the model. The solid black line depicts the averaged relationship between median filtered SIZ $V_{m}$ and IFR, while the dotted lines are one SD away from it. The red dashed line shows the best fitting power-law function ( $\alpha=125 \mathrm{~Hz}, V_{\max }=21.25 \mathrm{mV}$ and $n=1.52 ; r^{2}=0.95$ ).

dependence of the LGMD $I_{\mathrm{m}}$ on angular velocity documented in Figure $5 D$. The resulting change in $V_{\mathrm{m}}$, however, shows a much more gradual rise over time. Figure $7 C$ shows both the dendritic and SIZ $V_{\mathrm{m}}$ during looming, and replots the normalized excitatory synaptic conductance for comparison. Because the dendritic and SIZ $V_{\mathrm{m}}$ traces look very similar to one another, we conclude that the velocity tuning transformation occurs locally within the dendrites of the model, and that the SIZ $V_{\mathrm{m}}$ is a close reflection of the local dendritic $V_{\mathrm{m}}$. In Figure $7 D$, the excitatory conductance and resulting $V_{\mathrm{m}}$ at the SIZ are plotted against each other, showing that the transformation between the two signals can be described as a logarithmic compression. The light dashed lines show logarithmic fits to the curves and the inset shows that the relationship is correspondingly approximately linear when displayed on a logarithmic abscissa. The cause of the compression can be traced back to the reduction in driving force of synaptic currents as an increasing number of synapses are activated by the looming stimulus. This was verified by ascertaining that the local dendritic $V_{\mathrm{m}}$ approached the excitatory synaptic reversal potential of the model $(0 \mathrm{mV})$, and by carrying out simulations in which the reversal potential was increased, resulting in a predictable increase of the speed at which the $V_{\mathrm{m}}$ saturated.
We found that, in contrast to the striking compression of the total excitatory conductance's output, the total inhibitory conductance in the model was more subtly transformed. Since the reversal potential of the inhibitory inputs were only $10 \mathrm{mV}$ below the resting potential, we decided that the best way to measure the effect of inhibition on the model output would be to consider the difference in $V_{\mathrm{m}}$ of simulations run with and without inhibition. We call this $V_{\mathrm{m}}$ inhibitory influence and show its time course, along with stimulus angular size, in Figure 7E. This time course is consistent with that of feedforward inhibition observed experimentally at the level of the LGMD's firing rate (Gabbiani et al., 2005). The relationship between the two signals is shown in Figure $7 F$, and can be seen to be sigmoidal and well fit by a Weibull function. A similar plot produced for the total inhibitory input conductance shows an approximately quadratic relationship to the angular size (Fig. 7G), as expected from the sum of synaptic inputs generated when the looming stimulus encounters new facets. There is thus some compression of this inhibitory signal, as can be seen when comparing $G$ and $F$ in Figure 7, but certainly not nearly as strong as for excitation.

The observation that the excitatory synaptic conductance activated by a looming stimulus is compressed nearly logarithmically in 


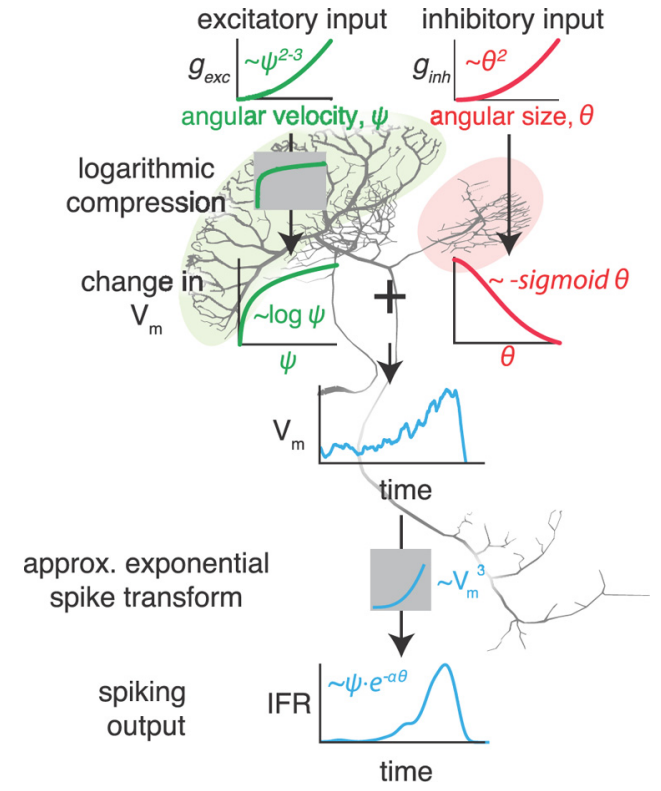

Figure 8. Schematic of the computation performed by the LGMD. During a looming stimulus, based on extrapolation from single-facet velocity tuning, the LGMD receives excitatory (left, green) inputs proportional to the object's angular velocity raised to a power of two to three. Our model inhibitory inputs (right, red) are approximately proportional to the square of the object's angular size, based on previous data. In our biophysical model, the excitatory signal undergoes a logarithmic compression in the dendritic tree, while the effect of inhibitory input on $V_{m}$ results in a sigmoidal dependence on angular size. Based on earlier results (bottom, cyan), the sum of these signals at the SIZ is then power-law transformed by the spike generation mechanism, which in vivo is close to an exponentiation, resulting in an output relationship that signals an angular threshold size through its firing rate peak.

the membrane potential of the model, while the inhibitory influence remains much closer to signaling angular size, fits the previously proposed model that excitatory and inhibitory inputs are effectively multiplied by an addition in logarithmic coordinates and subsequent approximate exponentiation via a nonlinear transformation at the spike initiation zone of the LGMD (Eq. 7; Gabbiani et al., 2002). Thus, we plotted the transformation between membrane potential at the SIZ and instantaneous firing rate to determine its computational role in the model (Fig. $7 H$ ). Fitting a power law to this transformation yielded an exponent, $n=1.57$, below the $n=3$ value observed experimentally (Gabbiani et al., 2002). A more expansive spike transform in the model, closer to that observed experimentally, would change the relative shapes of the speed-tuning functions for the model $V_{\mathrm{m}}$ and IFR, shown in Figure 6, but none of the other results. Additionally, because we used the peak LGMD membrane potential deflections to single-facet stimulation - not the firing rates- to constrain the strength of excitatory inputs, this relationship would have minimally affected other important model parameters. Thus, this difference with experimental data is unlikely to affect any of our main conclusions.

Overall, based on extrapolation from single-facet responses, our modeling suggests a plausible biophysical implementation of the computation performed by the LGMD (Fig. 8). The total excitatory input to the LGMD is expansively dependent on angular velocity but is logarithmically compressed by local reduction of driving force in the dendrites, resulting in membrane potential changes that are a saturating function of velocity. In the main dendritic trunk, this excitatory signal is summed with an inhibitory signal related to the stimulus' angular size; in our biophysical model, this inhibitory influence tracks angular size sublinearly. The resulting membrane potential is transformed by a final non- linear, expansive spike-thresholding step at the LGMD's SIZ. The output firing rate thus reflects a multiplication between the timevarying stimulus' angular velocity and a negative exponential of angular size.

\section{Discussion}

The simulations reported here were based on electrophysiological data documenting, either directly or indirectly, the activity of neurons at all successive stages of the excitatory pathway leading to the LGMD. This offered us a unique opportunity to understand how signals are encoded in real time starting from photoreceptors up to the final spiking output of this collision-detecting neuron. Broadly speaking, our results largely substantiate earlier hypotheses concerning the processing of excitatory and inhibitory signals within the LGMD. In particular, they identify where the postulated logarithmic transform of speed-dependent excitation takes place within the LGMD. They also offer a more nuanced perspective on the implementation of the multiplicative computation describing the LGMD's firing rate via a log-exp transform.

\section{Model validation}

Our results are based on a detailed compartmental model possessing several realistic features, including dendritic morphology, active conductance distribution, and spontaneous and evoked synaptic activity. In the process of model generation, a large body of constraining data has been used. Although it is undoubtedly the most accurate LGMD model to date, it nonetheless makes several simplifying assumptions. In addition to the lack of lateral interactions between excitatory inputs (see Materials and Methods), a further simplifying assumption lies in the modeling of feedforward inhibition. We abstracted the detailed structure of the LGMD's inhibitory dendritic branches to a simple synaptic connection at the location where the inhibitory dendrites contact the main dendritic trunk. Additionally, we have assumed the activation of inhibition to depend on the area covered by the stimulus, without explicitly modeling the dynamics and receptive field structure of the neurons presynaptic to the LGMD along the inhibitory pathway. Given the lack of precise information on how these neurons are activated by looming stimuli, our approach makes simple but plausible assumptions that will need to be verified experimentally. Thus, although we cannot draw any specific conclusions about the detailed activation time course of the inhibitory pathway to the LGMD during looming, we expect it will have little impact on our understanding of how the excitatory pathway is activated, which was the main focus of this work.

The model also used a passive dendritic tree. While there is some evidence for active conductances in the excitatory dendritic tree, such as a hyperpolarization activated current $\left(I_{\mathrm{h}}\right.$; Dewell and Gabbiani, unpublished observations), we do not yet know enough about their potential distribution and properties to warrant their inclusion. We expect that inclusion of $I_{\mathrm{h}}$ would, if anything, increase the signal compression observed through a depolarization-induced increase in membrane resistance. Voltagegated $\mathrm{K}^{+}$currents, if included, would lessen synaptic current saturation but would also hyperpolarize the membrane, likely preserving the logarithmic relationship between $g_{\text {exc }}$ and $V_{\mathrm{m}}$. Dendritic spikes have never been observed in the LGMD, thus voltage-gated sodium channels, if present, are at low densities and unlikely to significantly affect the compression observed. There are no detectable voltagegated calcium conductances in the dendrites (Peron and Gabbiani, 2009a). Despite the simplifications outlined above, the model repro- 
duced the main characteristics of the LGMD neuron's responses to looming stimuli reasonably well.

\section{Logarithmic transform}

The main thrust of our analysis was to simulate the membrane potential response of the LGMD to looming stimuli and infer the resulting representation of angular speed within the model. This enabled us to conclude that a logarithmic compression of angular speed is implemented between the conductances activating the excitatory portion of the LGMD's dendritic tree and the resulting, compound membrane potential recorded at the spike initiation zone of the neuron. This logarithmic compression is mainly caused by a saturation of the dendritic membrane potential toward the reversal potential of its excitatory synapses, which in turn can be traced back to the massive excitation impinging onto the LGMD neuron during a looming stimulus. Ultimately, this saturation requires individual synaptic currents to the LGMD to be sufficiently strong, which is supported by at least three observations. First and primarily, single-facet stimulation causes depolarization in the LGMD strong enough to elicit spikes (Peron et al., 2009; Jones and Gabbiani, 2010). The strength of those responses is precisely how we determined the excitatory synaptic conductance values of the model. Second and correspondingly, the LGMD also fires very early during looming stimuli, often when the stimulus initially appears and has an angular size of only few degrees. Third, since the looming stimulus sweeps across the visual field, it constantly triggers new synaptic inputs rather than restimulating old ones, removing short-term synaptic depression as a competing mechanism that would weaken synaptic input later during approach (O'Shea and Rowell, 1976). Additionally, logarithmic compression in the model was robust enough to withstand large changes in parameter values. In further simulations, we halved the model's membrane resistance to purposefully change the amount of saturation, and we still observed a similar logarithmic compression of excitatory input (data not shown).

This computation relates the time course of a compound conductance, distributed over the entire dendritic tree of the neuron, with the membrane potential at the spike initiation zone. Thus, it would have been difficult to identify the nature of this computation without a systematic experimental description of individual inputs to the LGMD and the subsequent simulations reported here. Future experiments recording the membrane potential within the smaller dendritic branches of LGMD's excitatory dendritic tree during the presentation of looming stimuli should enable the verification and further study of the characteristics of this logarithmic transform. A more detailed characterization would require recording directly from the LGMD's presynaptic afferents, an experiment that still lies beyond current technical means.

Logarithmic transforms have been postulated to take place within neurons in the past, most notably to implement the logarithmic compression of luminance information observed in vertebrate and invertebrate photoreceptors (Laughlin and Hardie, 1978). In this case, the logarithmic compression is most likely achieved within the phototransduction biochemical cascade, through a very different mechanism than that proposed here (van Hateren and Snippe, 2006). Similarly, synaptic saturation has been shown to play a role in motion detection in the fly, but the underlying mechanism is a local interaction between individual excitatory and inhibitory inputs, quite different from the global mechanism involving an extended dendritic tree described here (Single et al., 1997).
Our results raise the question of whether a similar logarithmic compression arises within the dendrites of looming sensitive neurons in other species that implement the same angular threshold computation as the LGMD. At present, very little is known about their specific intracellular properties and the representation of speed within their dendritic trees. Yet, this question could at least in principle be addressed in the frog optic tectum, for instance, since intracellular and patch-clamp recordings in conjunction with visual stimulation are feasible (Matsumoto et al., 1986; Svirskis et al., 2009). More generally and speculatively, a similar logarithmic compression of dendritic signals may also occur in the context of other neuron types. In visual cortex for instance, neuronal subpopulations may be predominantly excited within their dendritic trees in response to visual stimuli; if these excitatory inputs were widely spatially distributed and sufficiently strong, a similar dendritic integration scheme may ensue (Monier et al., 2003; Jia et al., 2010).

\section{Implication for other neural computations}

By systematically tracking the representation of angular speed along the excitatory pathway leading to the LGMD, we invariably found that it was encoded nonlinearly. More specifically, the responses of neural populations presynaptic to the LGMD were always better fit by power laws than by a linear function. Thus, although the model explaining the LGMD's firing rate to looming stimuli is based on a multiplication of angular velocity with a negative exponential of angular size, angular velocity itself was never directly represented within the neural populations presynaptic to the LGMD. Of course, this does not present an issue from the point of view of the LGMD's firing rate model and its log-exp transform formulation, because the logarithm of speed raised to a given power is simply equal to the logarithm of speed multiplied by the associated power's exponent: $\log \psi^{\mathrm{n}}=n \log \psi$. Nonetheless, this observation emphasizes that the most parsimonious implementation of a model based on its output representation may not turn out to be the one implemented within a specific neuronal network. Hence, other functional models whose outputs are described by the multiplication of two distinct terms, such as the Reichardt model currently investigated in the fruit fly (Clark et al., 2011; Eichner et al., 2011;), may never explicitly represent the two terms being multiplied.

\section{Concluding remarks}

Our experimental results and simulations identified the biophysical implementation of a logarithmic transform of angular speed between the excitatory synaptic conductance inputs to the LGMD and its membrane potential at the spike initiation zone. The synaptic mechanism involved is based on the saturation of the dendritic membrane potential during a looming stimulus as it approaches the reversal potential of synaptic conductances within the LGMD's excitatory dendritic tree. This is a collective computation, distributed over several thousand synapses, suggesting that dendritic trees are able to carry out sophisticated analog mathematical operations on their inputs before their conversion into spiking output. Similar computations may underlie sensory processing in other neurons across nervous systems.

\section{References}

Bazhenov M, Stopfer M, Rabinovich M, Huerta R, Abarbanel HD, Sejnowski TJ, Laurent G (2001) Model of transient oscillatory synchronization in the locust antennal lobe. Neuron 30:553-567.

Borst A, Euler T (2011) Seeing things in motion: models, circuits, and mechanisms. Neuron 71:974-994. 
Borst A, Haag J, Reiff DF (2010) Fly motion vision. Annu Rev Neurosci 33:49-70.

Clark DA, Bursztyn L, Horowitz MA, Schnitzer MJ, Clandinin TR (2011) Defining the computational structure of the motion detector in Drosophila. Neuron 70:1165-1177.

Eichner H, Joesch M, Schnell B, Reiff DF, Borst A (2011) Internal structure of the fly elementary motion detector. Neuron 70:1155-1164.

Fotowat H, Fayyazuddin A, Bellen HJ, Gabbiani F (2009) A novel neuronal pathway for visually guided escape in Drosophila melanogaster. J Neurophysiol 102:875-885.

Fotowat H, Harrison RR, Gabbiani F (2011) Multiplexing of motor information in the discharge of a collision detecting neuron during escape behaviors. Neuron 69:147-158.

Gabbiani F, Krapp HG (2006) Spike-frequency adaptation and intrinsic properties of an identified, looming-sensitive neuron. J Neurophysiol 96:2951-2962.

Gabbiani F, Krapp HG, Laurent G (1999) Computation of object approach by a wide-field, motion-sensitive neuron. J Neurosci 19:1122-1141.

Gabbiani F, Mo C, Laurent G (2001) Invariance of angular threshold computation in a wide-field looming-sensitive neuron. J Neurosci 21:314-329.

Gabbiani F, Krapp HG, Koch C, Laurent G (2002) Multiplicative computation in a visual neuron sensitive to looming. Nature 420:320-324.

Gabbiani F, Cohen I, Laurent G (2005) Time-dependent activation of feed-forward inhibition in a looming-sensitive neuron. J Neurophysiol 94:2150-2161.

Hassenstein B, Reichardt W (1956) Systemtheoretische Analyse der Zeit-, Reihenfolgen- und Vorzeichenauswertung bei der Bewegungsperzeption des Ruesselkaefers Chlorophanus. Z Naturforschg 11:513-524.

Hatsopoulos N, Gabbiani F, Laurent G (1995) Elementary computation of object approach by a wide-field visual neuron. Science 270:1000-1003.

Heiligenberg WF (1991) Neural nets in electric fish. Cambridge, MA. MIT.

Hines ML, Carnevale NT (1997) The NEURON simulation environment. Neural Comput 9:1179-1209.

Jia H, Rochefort NL, Chen X, Konnerth A (2010) Dendritic organization of sensory input to cortical neurons in vivo. Nature 464:1307-1312.

Jones PW, Gabbiani F (2010) Synchronized neural input shapes stimulus selectivity in a collision-detecting neuron. Curr Biol 20:2052-2057.

Jones PW, Gabbiani F (2012) Impact of neural noise on a sensory-motor pathway signaling impending collision. J Neurophysiol 107:1067-1079.

Juusola M, Uusitalo RO, Weckström M (1995) Transfer of graded potentials at the photoreceptor-interneuron synapse. J Gen Physiol 105:117-148.

Koch C. (1998) Biophysics of computation: information processing in single neurons. New York: Oxford UP.

Krapp HG, Gabbiani F (2005) Spatial distribution of inputs and local receptive field properties of a wide-field, looming sensitive neuron. J Neurophysiol 93:2240-2253.

Laughlin SB, Hardie RC (1978) Common strategies for light adaptation in the peripheral visual systems of fly and dragonfly. J Comp Physiol A Neuroethol Sens Neural Behav Physiol 128:319-340.

Liu YJ, Wang Q, Li B (2011) Neuronal responses to looming objects in the superior colliculus of the cat. Brain Behav Evol 77:193-205.

Matsumoto N, Schwippert WW, Ewert JP (1986) Intracellular activity of morphologically identified neurons of the grass frog's optic tectum in response to moving configurational visual stimuli. J Comp Physiol A Neuroethol Sens Neural Behav Physiol 159:721-739.

Monier C, Chavane F, Baudot P, Graham LJ, Frégnac Y (2003) Orientation and direction selectivity of synaptic inputs in visual cortical neurons: a diversity of combinations produces spike tuning. Neuron 37:663-680.

Nakagawa H, Hongjian K (2010) Collision-sensitive neurons in the optic tectum of the bullfrog, Rana catesbeiana. J Neurophysiol 104:2487-2499.

O'Shea M, Rowell CH (1976) The neuronal basis of a sensory analyser, the acridid movement detector system. II. response decrement, convergence, and the nature of the excitatory afferents to the fan-like dendrites of the LGMD. J Exp Biol 65:289-308.
O’Shea M, Rowell CH (1975) Protection from habituation by lateral inhibition. Nature 254:53-55.

O'Shea M, Rowell CHF, Williams J (1974) The anatomy of a locust visual interneurone; the descending contralateral movement detector. J Exp Biol 60:1-12.

Peña JL, Konishi M (2002) From postsynaptic potentials to spikes in the genesis of auditory spatial receptive fields. J Neurosci 22:5652-5658.

Peron S, Gabbiani F (2009a) Spike frequency adaptation mediates looming stimulus selectivity in a collision-detecting neuron. Nat Neurosci 12:318-326.

Peron SP, Gabbiani F (2009b) Role of spike-frequency adaptation in shaping neuronal response to dynamic stimuli. Biol Cybern 100:505-520.

Peron SP, Krapp HG, Gabbiani F (2007) Influence of electrotonic structure and synaptic mapping on the receptive field properties of a collisiondetecting neuron. J Neurophysiol 97:159-177.

Peron SP, Jones PW, Gabbiani F (2009) Precise subcellular input retinotopy and its computational consequences in an identified visual interneuron. Neuron 63:830-842.

Preuss T, Osei-Bonsu PE, Weiss SA, Wang C, Faber DS (2006) Neural representation of object approach in a decision-making motor circuit. J Neurosci 26:3454-3464.

Priebe NJ, Ferster D (2008) Inhibition, spike threshold, and stimulus selectivity in primary visual cortex. Neuron 57:482-497.

Priebe NJ, Mechler F, Carandini M, Ferster D (2004) The contribution of spike threshold to the dichotomy of cortical simple and complex cells. Nat Neurosci 7:1113-1122.

Rind FC, Simmons PJ (1992) Orthopteran DCMD neuron: a reevaluation of responses to moving objects. I. Selective responses to approaching objects. J Neurophysiol 68:1654-1666.

Rind FC, Simmons PJ (1998) Local circuit for the computation of object approach by an identified visual neuron in the locust. J Comp Neurol 395:405-415.

Rowell CH, O'Shea M, Williams JL (1977) The neuronal basis of a sensory analyser, the acridid movement detector system. IV. The preference for small field stimuli. J Exp Biol 68:157-185.

Schlotterer GR (1977) Response of the locust descending movement detector neuron to rapidly approaching and withdrawing visual stimuli. Can J Zool 55:1372-1376.

Single S, Haag J, Borst A (1997) Dendritic computation of direction selectivity and gain control in visual interneurons. J Neurosci 17:6023-6030.

Strausfeld NJ, Nässel DR. (1981) Neuroarchitectures serving compound eyes of crustacea and insects. In: Handbook of sensory physiology, Vol. VII/7B, pp 1-132. Berlin: Springer.

Sun H, Frost BJ (1998) Computation of different optical variables of looming objects in pigeon nucleus rotundus neurons. Nat Neurosci 1:296-303.

Svirskis G, Svirskiene N, Gutamaniene N (2009) An eye-tectum preparation allowing routine whole-cell recordings of neuronal responses to visual stimuli in the frog. J Neurosci Methods 180:22-28.

van den Beukel I, van Kleef RG, Zwart R, Oortgiesen M (1998) Physostigmine and acetylcholine differentially activate nicotinic receptor subpopulations in Locusta migratoria neurons. Brain Res 789:263-273.

van Hateren JH, Snippe HP (2006) Phototransduction in primate cones and blowfly photoreceptors: different mechanisms, different algorithms, similar response. J Comp Physiol A Neuroethol Sens Neural Behav Physiol 192:187-197.

Wei W, Feller MB. (2011) Organization and development of directionselective circuits in the retina. Trends Neurosci

Wilson M (1975) Angular sensitivity of light and dark adapted locust retinula cells. J Comp Physiol A Neuroethol Sens Neural Behav Physiol 97: 323-328.

Wu LQ, Niu YQ, Yang J, Wang SR (2005) Tectal neurons signal impending collision of looming objects in the pigeon. Eur J Neurosci 22:2325-2331. 